Bangladesh J. Plant Taxon. 25(2): 175-186, 2018 (December)

(C) 2018 Bangladesh Association of Plant Taxonomists

\title{
MERICARP MORPHOLOGY OF THE TRIBE SELINEAE (APIACEAE, APIOIDEAE) AND ITS TAXONOMIC IMPLICATIONS IN KOREA
}

\author{
Changyoung Lee ${ }^{1}$, Jinki Kim, Ashwini M. Darshetkar ${ }^{2}$, Ritesh Kumar Choudhary ${ }^{2}$, \\ SANG-Hong Park ${ }^{3}$, JoOngKu LeE ${ }^{4}$ and SANGHO Chol ${ }^{5}$ \\ International Biological Material Research Center, Korea Research Institute of Bioscience \& \\ Biotechnology, 125 Gwahak-ro, Yuseong-gu, Daejeon 34141, South Korea
}

Keywords: Mericarp surface characters; SEM; NMDS; UPGMA.

\begin{abstract}
Mericarp morphology of 24 taxa belonging to nine genera of the tribe Selineae (Family: Apiaceae) in Korea was studied by Scanning Electron Microscopy. UPGMA and NMDS analyses were performed based on 12 morphological characters. The mericarp surface characters like mericarp shape, rib number and shape, surface pattern, surface appendages and mericarp symmetry proved useful in distinguishing the genera of the tribe Selineae.
\end{abstract}

\section{Introduction}

The family Apiaceae comprises about 455 genera and is widely distributed across temperate regions of the world (Pimenov and Leonov, 1993). Many members of Apiaceae can be easily distinguished by umbellate inflorescence, fruits consisting of two one-seeded mericarps suspended from a split central column or carpophore and numerous minute epigynous flowers (Downie et al., 1998). Fruits of Apiaceae are known as cremocarps which in the dry state split into two mericarps. Each mericarp has a flat commissural and convex dorsal surface. Drude (1898) proposed a sound system of classification of Apiaceae with three subfamilies Hydrocotiloideae, Saniculoideae and Apioideae and 12 tribes. Apioideae is the largest subfamily consisting of 404 genera and about 2,935 species (Pimenov and Leonov, 1993) and can be distinguished from the other two subfamilies by the synapomorphies like the presence of compound umbels, well-developed vittae (secretory canals) and free carpophores. Selineae is one of the tribes of Apioideae and is represented by genera Angelica L., Carlesia Dunn., Cnidium Cusson ex Juss., Cymopterus Raf., Dystaenia Kitag., Glehnia F., Libanotis Haller ex Zinn, Ligusticum L., Peucedanum L. and Ostericum Hoffm. Species of the tribe Selineae are mostly distributed in China, Japan and Korea (Spalik et al., 2004).

Drude (1898) and many other workers like Calestani (1905), Koso-Poljansky (1916) considered fruit morphology and anatomy as important characters for classification of subfamily Apioideae. While Heywood (1971), Davis (1972), Cronquist (1982), doubted the validity of characters considered by Drude (1898) to diagnose evolutionary relationships. Later, various

\footnotetext{
${ }^{1}$ VNU University of Science, 334 Nguyen Trai, ThanhXuan, Hanoi, 100000, Vietnam.

${ }^{2}$ Biodiversity \& Palaeobiology Group (Plants), Agharkar Research Institute, G.G. Agarkar Road, Pune 411004, India.

${ }^{3}$ National Institute of Ecology, 1210, Geumgang-ro, Maseo-Myeon, Seocheon-gun, Chungnam, 325-813, Republic of Korea.

${ }^{4}$ Department of Environment and Forest Resources, Chungnam National University, Yuseong-gu, Daejeon, 34134, Republic of Korea.

${ }^{5}$ Corresponding author. E-mail: decoy0@kribb.re.kr
} 
molecular phylogenetic analyses using nrITS, and plastid markers like $r b c \mathrm{~L}, m a t \mathrm{~K}, r p o \mathrm{C} 1$ provided little supports to classifications based on anatomy and morphology of fruits (Kondo et al., 1996; Plunkett et al., 1996; Downie et al., 1998, 2001). In some analyses, the Arracacia clade arose within the Angelica clade (Plunkett et al., 1996; Downie et al., 1998) and Downie et al. (2001) suggested that the Arracacia clade may eventually be subsumed within the same. Both groups comprise many genera that were traditionally placed in tribe Peucedaneae subtribes Angelicinae and Ferulinae (Drude, 1898) or tribes Peucedaneae and Angeliceae (Pimenov and Leonov, 1993). Later, based on nrITS data Spalik et al. (2004) suggested that the Angelica and Arracacia clades form a strong monophyletic group. They described it as tribe Selineae which consists of 62 genera. However, various attempts to identify structural characters useful for delimiting the species under Selineae failed, even those based on molecular data (Ajani et al. 2008; Feng et al., 2009; Zhou et al., 2009).

Despite the consistent picture of relationships that has emerged in Apiaceae by molecular data, it has always been difficult to identify structural characters that could be used to classify and define the clades in Apiaceae with a major exception of fruit micromorphology and anatomy (Feng et al., 2009). Bagchi and Srivastava (1989) studied epicarp surfaces of some medicinally important Apiaceae members and concluded that the surface characters of epicarp of fruits are useful in distinguishing the species. Liu et al. (2006) studied the taxonomic value of fruit wing types in order Apiales suggesting that their structural data could complement DNA with observable features to recognize and circumscribe taxa. Similar studies were carried out by various workers (Lee et al., 2001; Spalik et al., 2001; Liu et al., 2006; Liao et al., 2013) which proved that the fruit micromorphology is useful in classifying and identifying taxa of Apiaceae. Lee $e t$ al. (1997), for the first time, studied seed morphology of eight medicinally important species of Apiaceae. Further, the importance of seed trait in the taxonomy of tribe Scandinae was also proved by molecular data (Lee et al., 2001). Recently, Liao et al. (2013) provided new insights into the phylogeny of Angelica and its allies based on nrDNA, cpDNA and morphological characters which included fruit anatomy and micromorphology.

In Korea, research on Selineae have been performed by various workers, but considerably less number of taxa were taken into account (Choi et al., 1998; Yoon, 2001; Koo and Kim, 2008). The present study was therefore conducted to analyze the usefulness of mericarp characters in distinguishing genera of the tribe Selineae.

\section{Material and Methods}

The present study includes 20 Korean taxa belonging to the tribe Selineae and four taxa representing the tribe Scandiceae. Seeds of collected specimens and herbarium specimens were used for the study and are deposited in KRIB and KBH. Details of voucher deposition are mentioned in Table 1. Fruits were carefully dissected without removing testa under dissecting microscope (Nikon, AZ100). Size and colour of the seeds were noted under dissecting microscope. For micromorphological observations, mature fruits were mounted on stubs using double sided adhesive tape. Each sample was coated with a thin layer $(20-40 \mathrm{~nm})$ of gold using Hitachi E-1010 sputter coater and examined at $20 \mathrm{KV}$ using a Hitachi S3400-N Scanning Electron Microscope.

For phenetic analysis, 12 qualitative characters of 24 Korean taxa were considered as mentioned in Table 2. The data was analyzed with the help of PAUP*4.0 (Swofford, 2001) to obtain a phenogram using the unweighted pair-group method with arithmetic mean (UPGMA) from the data matrix. Quantitative values were log-transformed to reduce the effect of zero values. The data was then subjected to non-metric multidimensional scaling (NMDS) following Quinn and Keough (2002). 
Table 1. Voucher information of the specimens examined in the study.

\begin{tabular}{|c|c|c|c|}
\hline Species & Place of collection & $\begin{array}{l}\text { Collectors and date } \\
\text { of collection }\end{array}$ & $\begin{array}{l}\text { Accession } \\
\text { number }\end{array}$ \\
\hline Angelica anomala Avé-Lall. & $\begin{array}{l}\text { Namwon-eup, Seogwipo-si, Jeju- } \\
\text { do, Korea }\end{array}$ & $\begin{array}{l}\text { Joongku Lee } \text { et al.; } \\
\text { 24.1.2008 }\end{array}$ & KRIB 0015965 \\
\hline $\begin{array}{l}\text { A. cartilaginomarginata } \\
\text { (Makino ex Y.Yabe) Nakai }\end{array}$ & $\begin{array}{l}\text { Deokchi-ri, Jucheon-myeon, } \\
\text { Namwon-si, Jeollabuk-do, Korea; } \\
\text { Sanbuk-ri, Unju-myeon, Wanju- } \\
\text { gun, Jeollabuk-do, Korea }\end{array}$ & $\begin{array}{l}\text { S.M. Lee \& H.Y. } \\
\text { Lee; } 21.10 .2009\end{array}$ & KRIB 0029410 \\
\hline $\begin{array}{l}\text { A. dahurica (Hoffm.) Benth. } \\
\text { \& Hook.f. ex Franch. \& Sav. }\end{array}$ & $\begin{array}{l}\text { Yeongpyeong-dong, Jeju-si, Jeju- } \\
\text { do, Korea }\end{array}$ & C.S. Kim; 7.8.2001 & KRIB 0001002 \\
\hline $\begin{array}{l}\text { A. decursiva (Miq.) Franch. } \\
\text { \& Sav. }\end{array}$ & $\begin{array}{l}\text { Sikjangsan, Sechon-dong, Dong- } \\
\text { gu, Daejeon, Korea }\end{array}$ & $\begin{array}{l}\text { Changyoung Lee; } \\
29.10 .2008\end{array}$ & KRIB 0020712 \\
\hline A. genuflexa Nutt. & $\begin{array}{l}\text { Oesam-ri, Bongseong-myeon, } \\
\text { Bonghwa-gun, Gyeongsangbuk-do, } \\
\text { Korea }\end{array}$ & $\begin{array}{l}\text { Geonrae Kim \& } \\
\text { Jinki Kim; } \\
\text { 17.0.2003 }\end{array}$ & KRIBBSD1040 \\
\hline A. gigas Nakai & $\begin{array}{l}\text { Daeamsan, Seohwa-myeon, Inje- } \\
\text { gun, Gangwon-do, Korea }\end{array}$ & $\begin{array}{l}\text { Joongku Lee } \text { et al.; } \\
6.10 .2004\end{array}$ & KRIB 0004085 \\
\hline A. grosseserrata Maxim. & $\begin{array}{l}\text { Unjangsan, Jeongcheon-myeon, } \\
\text { Jinan-gun, Jeollabuk-do, Korea }\end{array}$ & $\begin{array}{l}\text { Hyeong-Kyu Lee \& } \\
\text { Taejin Kim; } \\
\text { 19.7.2001 }\end{array}$ & KRIB 0001031 \\
\hline A. japonica A. Gray & $\begin{array}{l}\text { Geomundo, Deokchon-ri, Samsan- } \\
\text { myeon, Yeosu-si, Jeollanam-do, } \\
\text { Korea }\end{array}$ & $\begin{array}{l}\text { Jinki Kim \& } \\
\text { Changyoung Lee; } \\
\text { 2.2.2009 }\end{array}$ & KRIB 0029071 \\
\hline A. polymorpha Maxim. & $\begin{array}{l}\text { Oebang-ri, Sudong-myeon, } \\
\text { Namyangju-si, Gyeonggi-do, Korea }\end{array}$ & $\begin{array}{l}\text { J.H. Kim et al.; } \\
\text { 12.11.2007 }\end{array}$ & KRIB 0013530 \\
\hline A. tenuissima Nakai & $\begin{array}{l}\text { Geumdaebong, Gohan-eup, } \\
\text { Jeongseon-gun, Gangwon-do, } \\
\text { Korea }\end{array}$ & $\begin{array}{l}\text { Hyeong-Kyu Lee \& } \\
\text { Taejin Kim; } \\
\text { 12.9.2001 }\end{array}$ & KRIB 0001020 \\
\hline $\begin{array}{l}\text { Anthriscus sylvestris (L.) } \\
\text { Hoffm. }\end{array}$ & $\begin{array}{l}\text { Ulleungdo, Ulleung-gun, } \\
\text { Gyeongsangbuk-do, Korea }\end{array}$ & $\begin{array}{l}\text { Shinho Kang et al.; } \\
\text { 14.6.2006 }\end{array}$ & KRIB 0006619 \\
\hline Cnidium japonicum Miq. & $\begin{array}{l}\text { Susan-ri, Seongsan-eup, Seogwipo- } \\
\text { si, Jeju-do, Korea }\end{array}$ & $\begin{array}{l}\text { C.S. Kim et al; } \\
15.11 .2006\end{array}$ & KRIB 0007560 \\
\hline C. monnieri (L.) Cusson & Goyang-si, Gyeonggi-do, Korea & $\begin{array}{l}\text { W.K. Paik; } \\
10.11 .2001\end{array}$ & KRIB 0010062 \\
\hline $\begin{array}{l}\text { Cymopterus melanotilingia } \\
\text { (H. Boissieu) C.Y. Yoon }\end{array}$ & $\begin{array}{l}\text { Gayasan, Gaya-myeon, Hapcheon- } \\
\text { gun, Gyeongsangnam-do, Korea }\end{array}$ & $\begin{array}{l}\text { S.M. Lee \& H.Y. } \\
\text { Lee; } 1.11 .2006\end{array}$ & KRIB 0006906 \\
\hline $\begin{array}{l}\text { Dystaenia takesimana } \\
\text { (Nakai) Kitag. }\end{array}$ & $\begin{array}{l}\text { Ulleungdo, Ulleung-gun, } \\
\text { Gyeongsangbuk-do, Korea }\end{array}$ & $\begin{array}{l}\text { Taejin Kim; } \\
13.10 .2000\end{array}$ & KRIB 0003115 \\
\hline $\begin{array}{l}\text { Glehnia littoralis (A. Gray) } \\
\text { F. Schmidtex Miq. }\end{array}$ & $\begin{array}{l}\text { Saekdal-dong, Seogwipo-si, Jeju- } \\
\text { do, Korea }\end{array}$ & $\begin{array}{l}\text { J.H. Kim et al.; } \\
\text { 19.6.2002 }\end{array}$ & KRIB 0011773 \\
\hline $\begin{array}{l}\text { Libanotis seseloides (Fisch. } \\
\text { \& C.A. Mey. ex Turcz.) } \\
\text { Turcz. }\end{array}$ & $\begin{array}{l}\text { Baekunsan, Mitan-myeon, } \\
\text { Pyeongchang-gun, Gangwon-do, } \\
\text { Korea }\end{array}$ & $\begin{array}{l}\text { Jinki Kim \& } \\
\text { Sanghong Park; } \\
\text { 20.10.2005 }\end{array}$ & KBH1053185 \\
\hline $\begin{array}{l}\text { Ligusticum tachiroei (Franch. } \\
\text { \& Sav.) M. Hiroe \& } \\
\text { Constance }\end{array}$ & $\begin{array}{l}\text { Jeombongsan, Girin-myeon, Inje- } \\
\text { gun, Gangwon-do, Korea }\end{array}$ & $\begin{array}{l}\text { Jinki Kim \& } \\
\text { Sanghong Park; } \\
\text { 14.10.2004 }\end{array}$ & KBH1032523 \\
\hline $\begin{array}{l}\text { Osmorhiza aristata (Thunb.) } \\
\text { Rydb. }\end{array}$ & $\begin{array}{l}\text { Juwangsan, Budong-myeon, } \\
\text { Cheongsong-gun, Gyeongsangbuk- } \\
\text { do, Korea }\end{array}$ & $\begin{array}{l}\text { G.Y. Chung et al.; } \\
\text { 22.8.2002 }\end{array}$ & KRIB 0011782 \\
\hline
\end{tabular}


Table 1 Contd.

\begin{tabular}{|c|c|c|c|}
\hline Species & Place of collection & $\begin{array}{l}\text { Collectors and date } \\
\text { of collection }\end{array}$ & $\begin{array}{l}\text { Accession } \\
\text { number }\end{array}$ \\
\hline $\begin{array}{l}\text { Ostericum sieboldii (Miq.) } \\
\text { Nakai }\end{array}$ & $\begin{array}{l}\text { Yongsan-ri, Doam-myeon, } \\
\text { Pyeonchang-gun, Gangwon-do, } \\
\text { Korea }\end{array}$ & $\begin{array}{l}\text { W.K. Paik; } \\
19.10 .2000\end{array}$ & KRIB 0010040 \\
\hline Peucedanum japonicum Thunb. & $\begin{array}{l}\text { Oeyeondo, Ocheon-myeon, } \\
\text { Boryeong-si, Chungcheongnam-do, } \\
\text { Korea }\end{array}$ & $\begin{array}{l}\text { Changyoung Lee; } \\
2.10 .2009\end{array}$ & KBH1259205 \\
\hline $\begin{array}{l}\text { P. terebinthaceum (Fisch. ex } \\
\text { Trevir.) Ledeb. }\end{array}$ & $\begin{array}{l}\text { Daejin-ri, Yeonghae-myeon, } \\
\text { Yeongdeok-gun, Gyeongsangbuk- } \\
\text { do, Korea }\end{array}$ & $\begin{array}{l}\text { J.H. Kim et al.; } \\
\text { 12.10.2001 }\end{array}$ & KRIB 0010050 \\
\hline Torilis japonica (Houtt.) DC. & $\begin{array}{l}\text { Cheondeungsan, Sancheok-myeon, } \\
\text { Chungju-si, Chungcheongbuk-do, } \\
\text { Korea }\end{array}$ & $\begin{array}{l}\text { G.Y. Chung et al.; } \\
5.8 .2002\end{array}$ & KRIB 0011785 \\
\hline T. scabra (Thunb.) DC. & $\begin{array}{l}\text { Sanbangsan, Sagye-ri, Andeok- } \\
\text { myeon, Seogwipo-si, Jeju-do, } \\
\text { Korea }\end{array}$ & $\begin{array}{l}\text { Taejin Kim et al.: } \\
\text { 28.5.2002 }\end{array}$ & KRIB 0002834 \\
\hline
\end{tabular}

Table 2. Codes used for for mericarp character analysis.

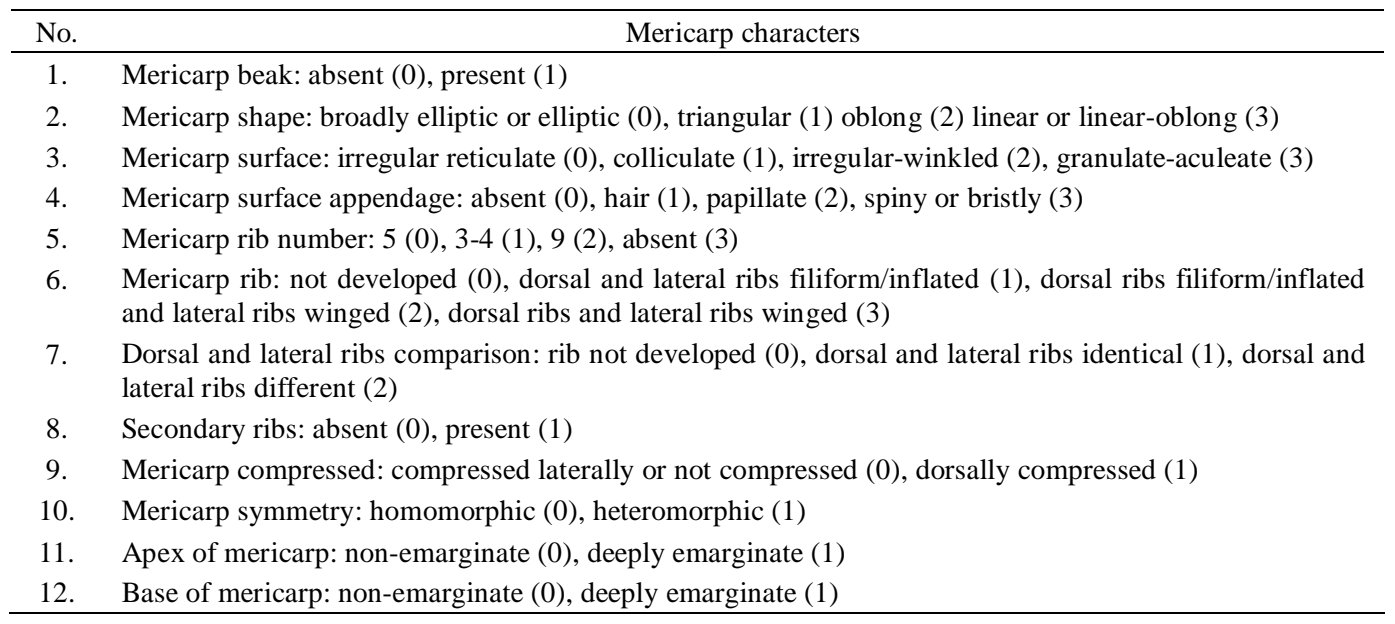

Stress values resulting from NMDS reflect the match between the pairwise differences originally calculated between the species and the pairwise distances in the ordination. Lower stress values indicate a better match (Quinn and Keough, 2002). This statistical analysis was performed using PAST (Hammer et al., 2001). Names and authorities of the species are as per The Plant List (2013). Terminology to describe the seed coat and seed surface sculpturing follows Harris and Harris (1994), Stearn (1992), and Webb and Simpson (2001).

\section{Results and Discussion}

The results obtained from the study are summarized in Table 3. Photomicrographs of mericarps and mericarp surfaces are presented in Figures 1 and 2, respectively. In the tribe Selineae, large variation can be seen in sizes of mericarps. The length of mericarp varies from 
$2.7 \pm 0.2$ to $11.4 \pm 0.6 \mathrm{~mm}$ (Fig. 3), while the width of mericarp varies from $1.4 \pm 0.2$ to $9.3 \pm 0.4 \mathrm{~mm}$ (Fig. 4). Seed size of Glehnia littoralis is highly divergent than other species included in the study (Figs 3 \& 4). Three different shapes of mericarp were observed during our study. The genera Ligusticum and Libanotis are characterized by oblong mericarps, the genus Glehnia is characterized by triangular mericarp, while broadly elliptic-elliptic mericarp was observed in the genera Cnidium, Angelica, Ostericum, Cymopterus and Peucedanum. In all species of Selineae, mericarps are symmetrical with five ribs except in Cymopterus melanotilingia, where asymmetric mericarps with three or four ribs were observed.
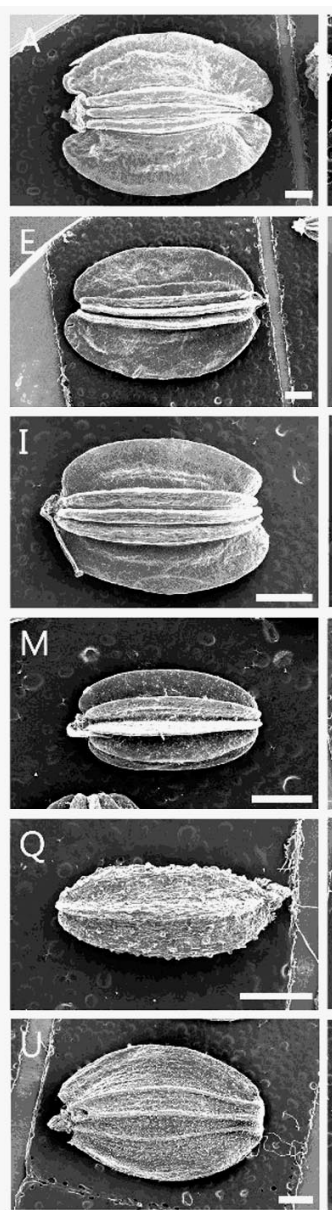
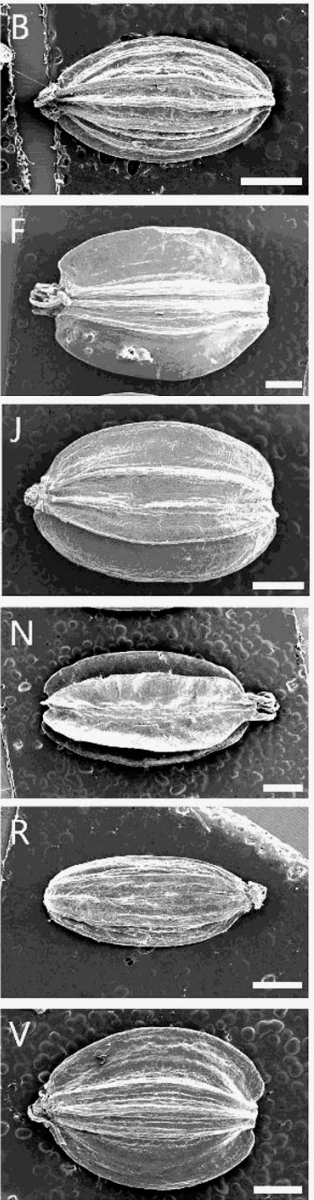
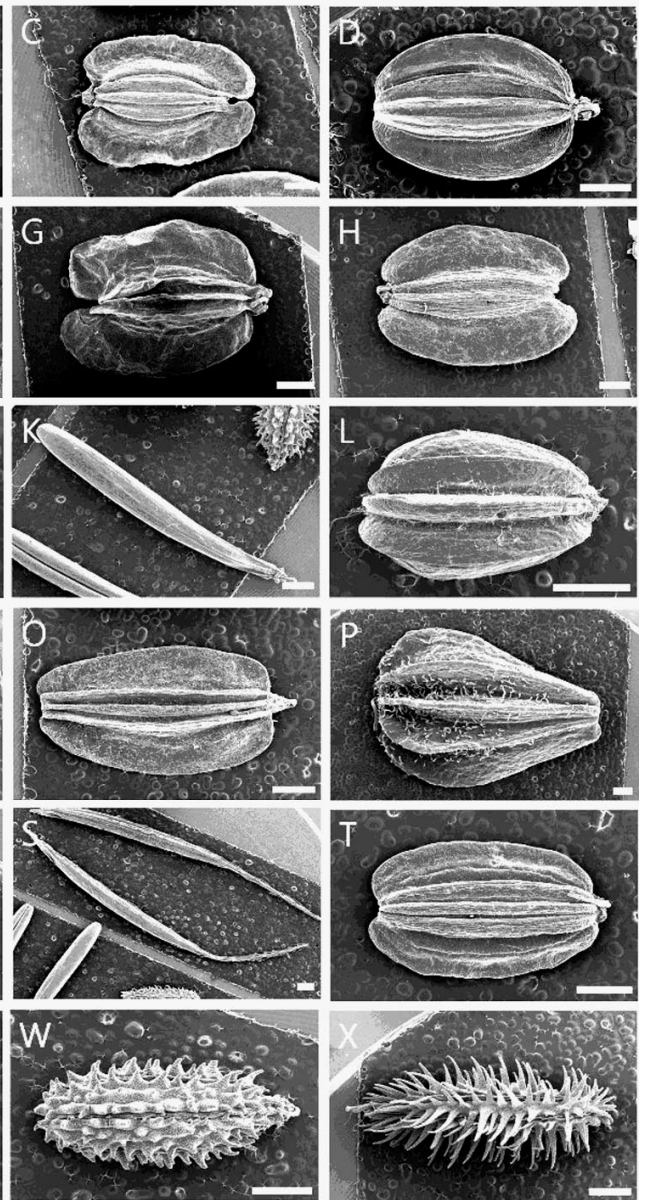

Fig. 1. SEM images of mericarps of 24 taxa of Selineae: A. Angelica anomala; B. A. cartilaginomarginata; C. A. dahurica; D. A. decursiva; E. A. genuflexa; F. A. gigas; G. A. grosseserrata; H. A. japonica; I. A. polymorpha; J. A. tenuissima; K. Anthriscus sylvestris; L. Cnidium japonicum; M. C. monnieri; N. Cymopterus melanotilingia; O. Dystaenia takesimana; P. Glehnia littoralis; Q. Libanotis seseloides; R. Ligusticum tachiroei; S. Osmorhiza aristata; T. Ostericum sieboldii; U. Peucedanum japonicum; V. P. terebinthaceum; W. Torilis japonica; X. T. scabra. Scale bar: $1 \mathrm{~mm}$.

Three different surface patterns characterize Selineae mericarps. The irregular reticulate surface pattern was observed in Cnidium, Dystaenia, Glehnia and Angelica cartilaginomarginata, A. genuflexa, A. japonica, A. anomala, A. gigas, and Peucedanum terebinthaceum. While 
Ligusticum, Ostericum, Cymopterus, Angelica tenuissima, A. polymorpha, A. dahurica, and A. decursiva showed colliculate surface. The irregular-wrinkled surface pattern was observed in Libanotis seseloides and Peucedanum japonicum. Papillate surface pattern characterizes the genus Libanotis. Hairs were observed on mericarps of Glehnia littoralis and Peucedanum japonicum (Fig. 2). In all species of Selineae employed in this study, mericarps are symmetrical with five ribs except in Cymopterus melanotilingia, where asymmetric mericarps with three or four ribs were observed.
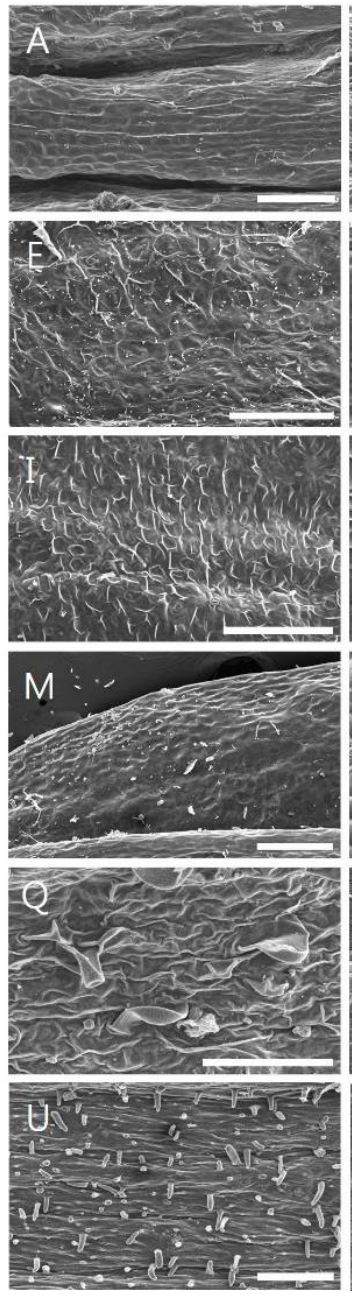
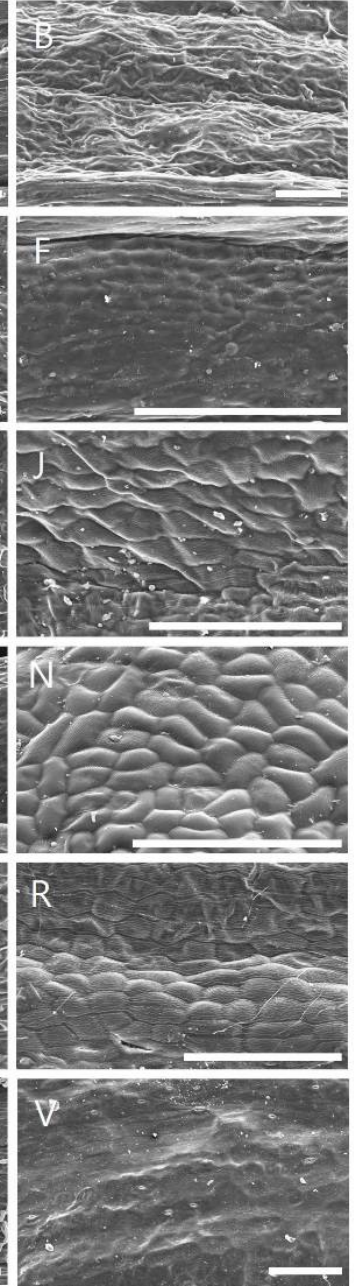
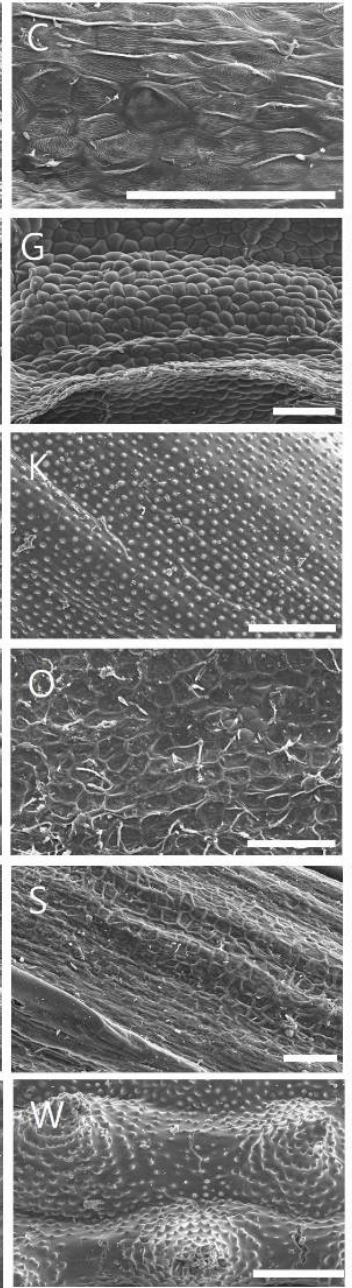
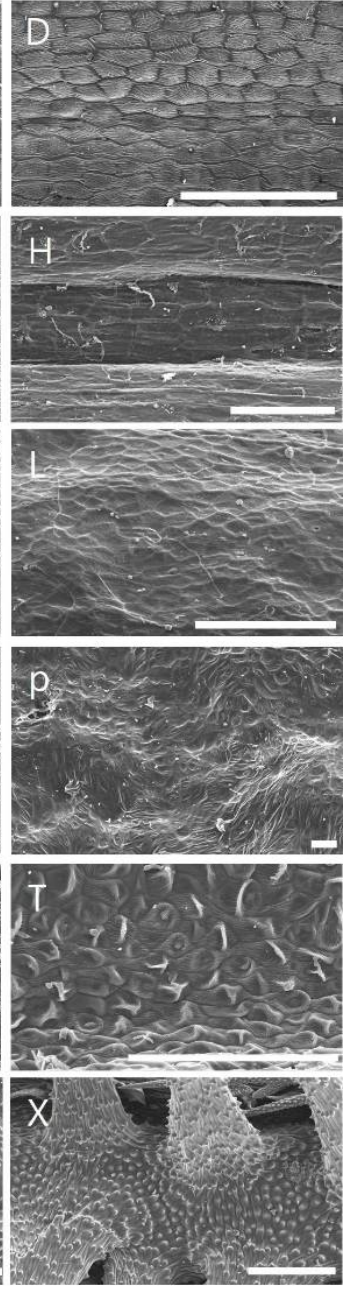

Fig. 2. Mericarp surface characters of 24 taxa of Selineae: A. Angelica anomala; B. A. cartilaginomarginata; C. A. dahurica; D. A. decursiva; E. A. genuflexa; F. A. gigas; G. A. grosseserrata; H. A. japonica; I. A. polymorpha; J. A. tenuissima; K. Anthriscus sylvestris; L. Cnidium japonicum; M. C. monnieri; N. Cymopterus melanotilingia; O. Dystaenia takesimana; P. Glehnia littoralis; Q. Libanotis seseloides; R. Ligusticum tachiroei; S. Osmorhiza aristata; T. Ostericum sieboldii; U. Peucedanum japonicum; V. P. terebinthaceum; W. Torilis japonica; X. T. scabra. Scale bar: $200 \mu \mathrm{m}$. 
Table 3. Morphological characters of mericarp of 24 species in the tribe Selineae.

\begin{tabular}{|c|c|c|c|c|c|c|c|}
\hline \multirow[t]{2}{*}{ Species } & \multirow{2}{*}{$\begin{array}{l}\text { Length } \\
(\mathrm{mm})\end{array}$} & \multirow{2}{*}{$\begin{array}{l}\text { Width } \\
(\mathrm{mm})\end{array}$} & \multirow[t]{2}{*}{ Mericarp shape } & \multirow{2}{*}{$\begin{array}{l}\text { Mericarp } \\
\text { surface }\end{array}$} & \multirow{2}{*}{$\begin{array}{l}\text { Appen- } \\
\text { dage }\end{array}$} & \multicolumn{2}{|c|}{ Ribs } \\
\hline & & & & & & Lateral & Dorsal \\
\hline Anthriscus sylvestris & $8.2 \pm 0.5$ & $1.0 \pm 0.1$ & linear-oblong & $\begin{array}{l}\text { granulate- } \\
\text { aculeate }\end{array}$ & Absent & Absent & Absent \\
\hline A. anomala & $5.9 \pm 0.5$ & $5.0 \pm 0.4$ & broadly elliptic & reticulate & Absent & winged & inflated \\
\hline $\begin{array}{l}\text { A. cartilagino- } \\
\text { marginata }\end{array}$ & $3.3 \pm 0.3$ & $1.9 \pm 0.3$ & $\begin{array}{l}\text { broadly elliptic } \\
\text { or elliptic }\end{array}$ & $\begin{array}{l}\text { irregular- } \\
\text { reticulate }\end{array}$ & Absent & winged & inflated \\
\hline A. dahurica & $5.8 \pm 0.5$ & $5.2 \pm 0.5$ & broadly elliptic & colliculate & Absent & winged & inflated \\
\hline A. decursiva & $4.8 \pm 0.6$ & $3.1 \pm 0.4$ & $\begin{array}{l}\text { broadly elliptic } \\
\text { or elliptic }\end{array}$ & colliculate & Absent & winged & inflated \\
\hline A. genuflexa & $6.2 \pm 0.6$ & $4.6 \pm 0.4$ & broadly elliptic & $\begin{array}{l}\text { irregular- } \\
\text { reticulate }\end{array}$ & Absent & winged & inflated \\
\hline A. gigas & $6.4 \pm 0.5$ & $4.8 \pm 0.4$ & broadly elliptic & $\begin{array}{l}\text { irregular- } \\
\text { reticulate }\end{array}$ & Absent & winged & inflated \\
\hline A. japonica & $10.1 \pm 0.6$ & $6.1 \pm 0.3$ & $\begin{array}{l}\text { broadly elliptic } \\
\text { or elliptic }\end{array}$ & reticulate & Absent & winged & inflated \\
\hline A. polymorpha & $4.2 \pm 0.3$ & $3.1 \pm 0.2$ & broadly elliptic & colliculate & Absent & winged & inflated \\
\hline Angelica grosseserrata & $5.6 \pm 0.7$ & $4.6 \pm 0.8$ & broadly elliptic & colliculate & Absent & winged & inflated \\
\hline A. tenuissima & $5.4 \pm 0.5$ & $3.6 \pm 0.6$ & $\begin{array}{l}\text { broadly elliptic } \\
\text { or elliptic }\end{array}$ & colliculate & Absent & winged & inflated \\
\hline Cnidium japonicum & $3.0 \pm 0.2$ & $2.0 \pm 0.2$ & $\begin{array}{l}\text { Broadly elliptic } \\
\text { or elliptic }\end{array}$ & reticulate & Absent & winged & winged \\
\hline C. monnieri & $2.9 \pm 0.2$ & $1.8 \pm 0.2$ & $\begin{array}{l}\text { broadly elliptic } \\
\text { or elliptic }\end{array}$ & $\begin{array}{l}\text { irregular- } \\
\text { reticulate }\end{array}$ & Absent & winged & winged \\
\hline $\begin{array}{l}\text { Cymopterus } \\
\text { melanotilingia }\end{array}$ & $6.0 \pm 0.3$ & $3.1 \pm 0.2$ & elliptic & colliculate & Absent & winged & winged \\
\hline Dystaenia takesimana & $5.7 \pm 0.6$ & $3.2 \pm 0.4$ & $\begin{array}{l}\text { broadly elliptic } \\
\text { or elliptic }\end{array}$ & reticulate & Absent & winged & winged \\
\hline Glehnia littoralis & $11.4 \pm 0.6$ & $9.3 \pm 0.4$ & triangular & $\begin{array}{l}\text { irregular- } \\
\text { reticulate }\end{array}$ & hairy & winged & winged \\
\hline Libanotis seseloides & $2.7 \pm 0.2$ & $1.4 \pm 0.2$ & oblong & $\begin{array}{l}\text { irregular- } \\
\text { winkled }\end{array}$ & papillate & inflated & inflated \\
\hline Ligusticum tachiroei & $4.0 \pm 0.2$ & $2.0 \pm 0.2$ & oblong & colliculate & Absent & inflated & inflated \\
\hline Osmorhiza aristata & $17.6 \pm 1.0$ & $1.0 \pm 0.1$ & linear & reticulate & bristly & filiform & filiform \\
\hline Ostericum sieboldii & $3.8 \pm 0.3$ & $2.6 \pm 0.2$ & $\begin{array}{l}\text { broadly elliptic } \\
\text { or elliptic }\end{array}$ & colliculate & Absent & winged & inflated \\
\hline Peucedanum japonicum & $5.1 \pm 0.5$ & $2.1 \pm 0.2$ & elliptic & $\begin{array}{l}\text { irregular- } \\
\text { winkled }\end{array}$ & hairy & winged & filiform \\
\hline P. terebinthaceum & $4.6 \pm 0.2$ & $3.3 \pm 0.2$ & broadly elliptic & $\begin{array}{l}\text { irregular- } \\
\text { reticulate }\end{array}$ & Absent & winged & inflated \\
\hline Torilis japonica & $3.8 \pm 0.3$ & $1.4 \pm 0.1$ & oblong & $\begin{array}{l}\text { granulate- } \\
\text { aculeate }\end{array}$ & spiny & filiform & filiform \\
\hline T. scabra & $5.9 \pm 0.5$ & $1.2 \pm 0.2$ & oblong & $\begin{array}{l}\text { granulate- } \\
\text { aculeate }\end{array}$ & spiny & filiform & filiform \\
\hline
\end{tabular}

We found three different mericarp rib shapes in Selineae. Ligusticum tachiroei and Libanotis seseloides have dorsal and lateral ribs which are filiform or inflated. The genera Angelica, Ostericum and Peucedanum are characterized by filliform or inflated dorsal ribs and winged lateral ribs, while the genera Cnidium, Cymopterus, Glehnia and Dystaenia showed winged dorsal and lateral ribs. 


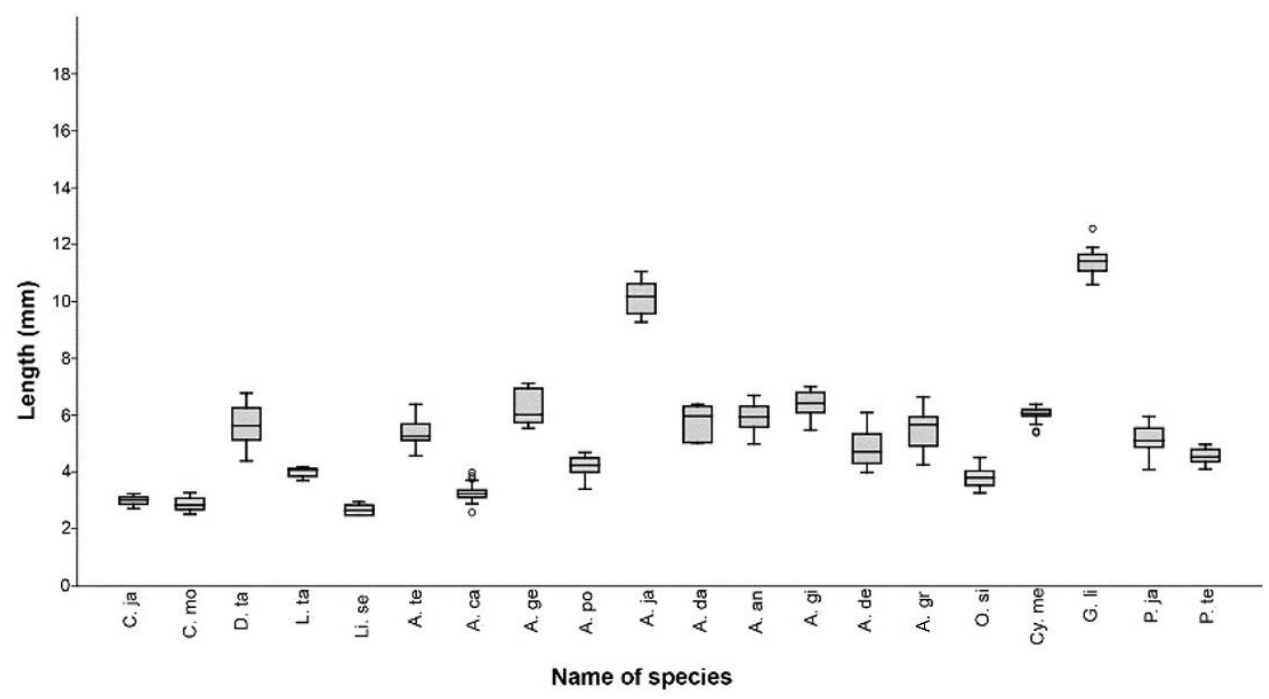

Fig. 3. Length of seeds summarized in Boxplot. The boxes represent the second and third quartiles, and the vertical line within each of the boxes represents the median. Outliers are identified with asterisks (C. ja: Cnidium japonicum; C. mo: C. monnieri; D. ta: Dystaenia takesimana; L. ta: Ligusticum tachiroei; Li. se: Libanotis seseloides; A. te: Angelica tenuissima; A. ca: A. cartilaginomarginata; A. ge: A. genuflexa; A. po: A. polymorpha; A. ja: A. japonica; A. da: A. dahurica; A. an: A. anomala; A. gi: A. gigas; A. de: A. decursiva; A. gr: A. grosseserrata; O. si: Ostericum sieboldii; Cy. me: Cymopterus melanotilingia; G. li: Glehnia littoralis; P. ja: Peucedanum japonicum; P. te: Peucedanum terebinthaceum).

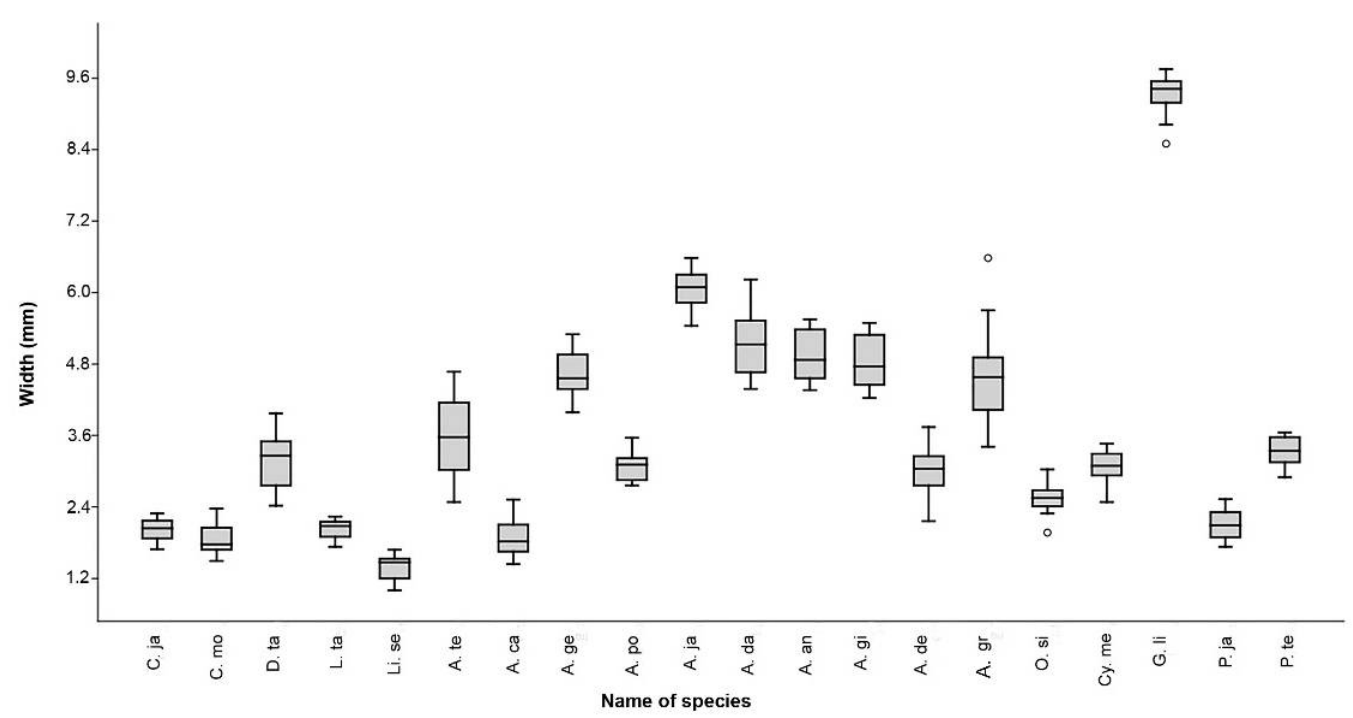

Fig. 4. Width of seeds summarized in Boxplot. The boxes represent the second and third quartiles, and the vertical line within each of the boxes represents the median. Outliers are identified with asterisks. (C. ja: Cnidium japonicum; C. mo: C. monnieri; D. ta: Dystaenia takesimana; L. ta: Ligusticum tachiroei; Li. se: Libanotis seseloides; A. te: Angelica tenuissima; A. ca: A. cartilaginomarginata; A. ge: A. genuflexa; A. po: A. polymorpha; A. ja: A. japonica; A. da: A. dahurica; A. an: A. anomala; A. gi: A. gigas; A. de: A. decursiva; A. gr: A. grosseserrata; O. si: Ostericums ieboldii; Cy. me: Cymopterus melanotilingia; G. li: Glehnia littoralis; P. ja: Peucedanum japonicum; P. te: Peucedanum terebinthaceum). 
Emarginated mericarp apex was observed in Angelica japonica, A. dahurica, and A. anomala, while in other studied species mericarp apex was non-emarginate. Deeply emarginated mericarp base was observed in Angelica genuflexa, A. polymorpha, A. japonica, A. dahurica, A. anomala, A. grosseserrata, Ostericum sieboldii, Cymopterus melanotilingia and Glehnia littoralis, while in other species non-emarginated mericarp base was noticed.

\section{Phenetic analysis}

UPGMA phenogram was evaluated to identify characters that distinguish the genera of Apioideae. The analysis revealed seven major groups. The genus Glehnia can be distinguished from other genera of Selineae by triangular mericarp and hairy mericarp surface (Pairwise distance 2.474). Ligusticum tachiroei and Libanotis seseloides appear to be sister groups as oblong mericarps characterize them. Both the species can be distinguished by mericarp surface pattern and appendages. Ligusticum tachiroei has colliculate surface pattern and lacks appendages (pairwise distance 1.000), whereas Libanotis seseloides has irregularly wrinkled and papillate surface. Cymopterus melanotilingia can be distinguished from other genera like Peucedanum, Angelica, Ostericum, Cnidium and Dystaenia as it has asymmetric mericarp and rib number 3-4 (pairwise distance 2.188). Cnidium and Dystaenia appear to be sister groups (pairwise distance 1.064) and are characterized by winged dorsal and lateral ribs. Based on UPGMA analysis of mericarp surface characters, the genera Glehnia, Cymopterus, Ligusticum, Libanotis, Cnidium and Dystaenia can be distinguished, however, Angelica, Peucedanum and Ostericum cannot be distinguished. NMDS analysis of Bray-Curtis similarity coefficients (Fig. 5) calculated from 12 morphological characters showed similar separation of species as in UPGMA (Fig. 6).

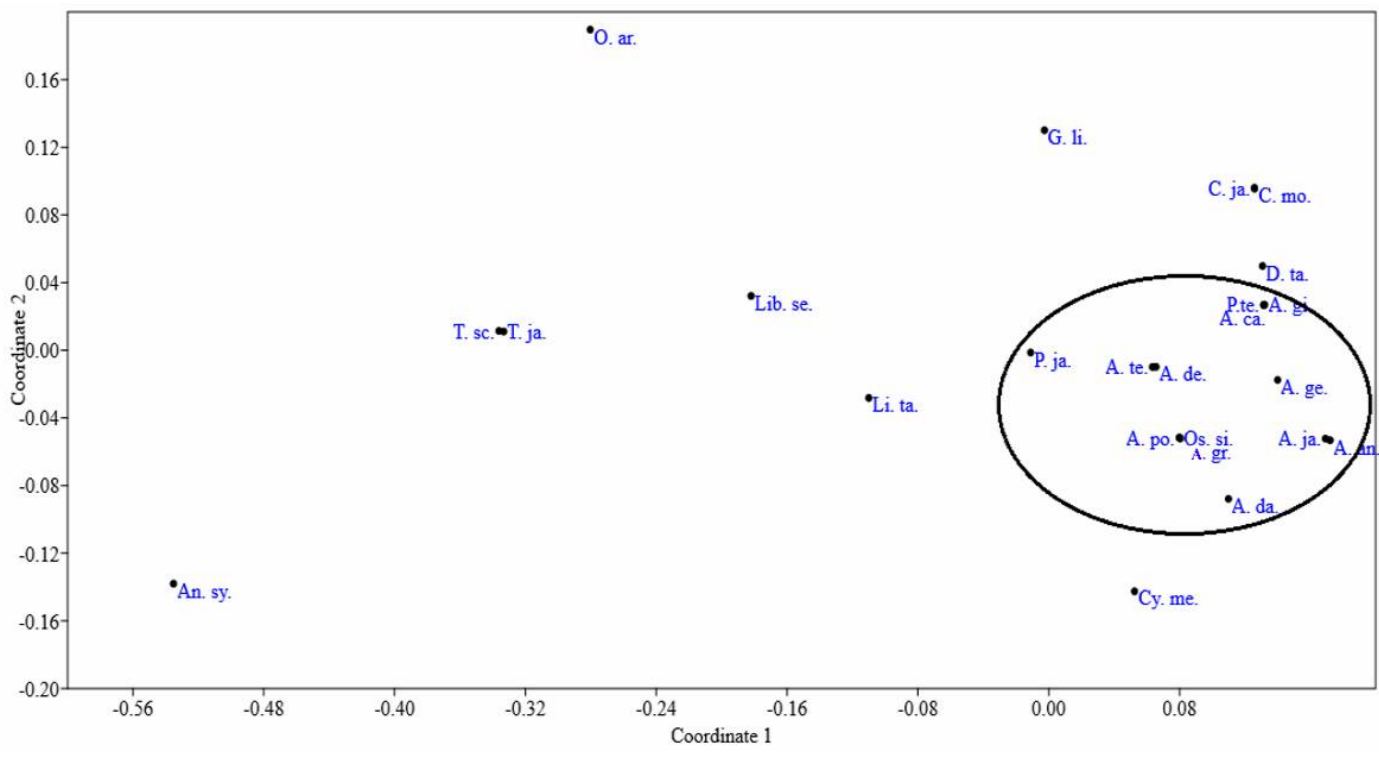

Fig. 5. Non-metric multidimensional scaling (NMDS) using Bray-Curtis similarity index of 12 morphological characters. (C. ja: Cnidium japonicum; C. mo: C. monnieri; D. ta: Dystaenia takesimana; L. ta: Ligusticum tachiroei; Li. se: Libanotis seseloides; A. te: Angelica tenuissima; A. ca: A. cartilaginomarginata; A. ge: A. genuflexa; A. po: A. polymorpha; A. ja: A. japonica; A. da: A. dahurica; A. an: A. anomala; A. gi: A. gigas; A. de: A. decursiva; A. gr: A. grosseserrata; O. si: Ostericum sieboldii; Cy. me: Cymopterus melanotilingia; G. li: Glehnia littoralis; P. ja: Peucedanum japonicum; P. te: Peucedanum terebinthaceum). 
To conclude, characters like mericarp shape, rib number, and shape, surface pattern, surface appendages and mericarp symmetry can be considered as useful traits to distinguish the genera of tribe Selineae. These results are in congruence with the molecular systematic study of Angelica and allied genera based on nuclear DNA sequences (Feng et al., 2009).

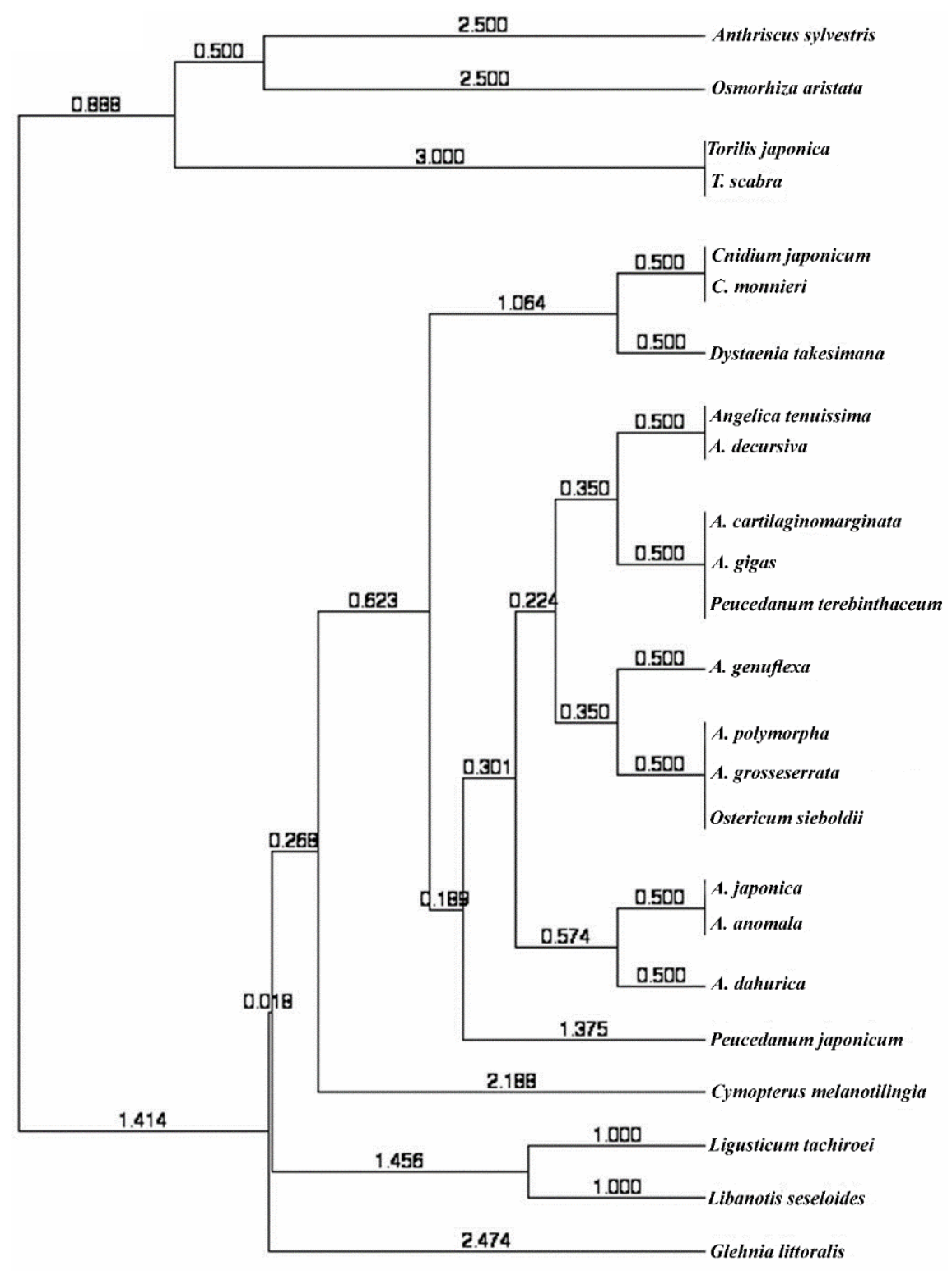

Fig. 6. UPGMA phenogram of tribe Selineae and Scandiceae based on the 12 morphological characters. Values above lines indicate pairwise distances.

A taxonomic key based on the mericarp characters has been provided below for the easy identification of Selineae members in Korea. 


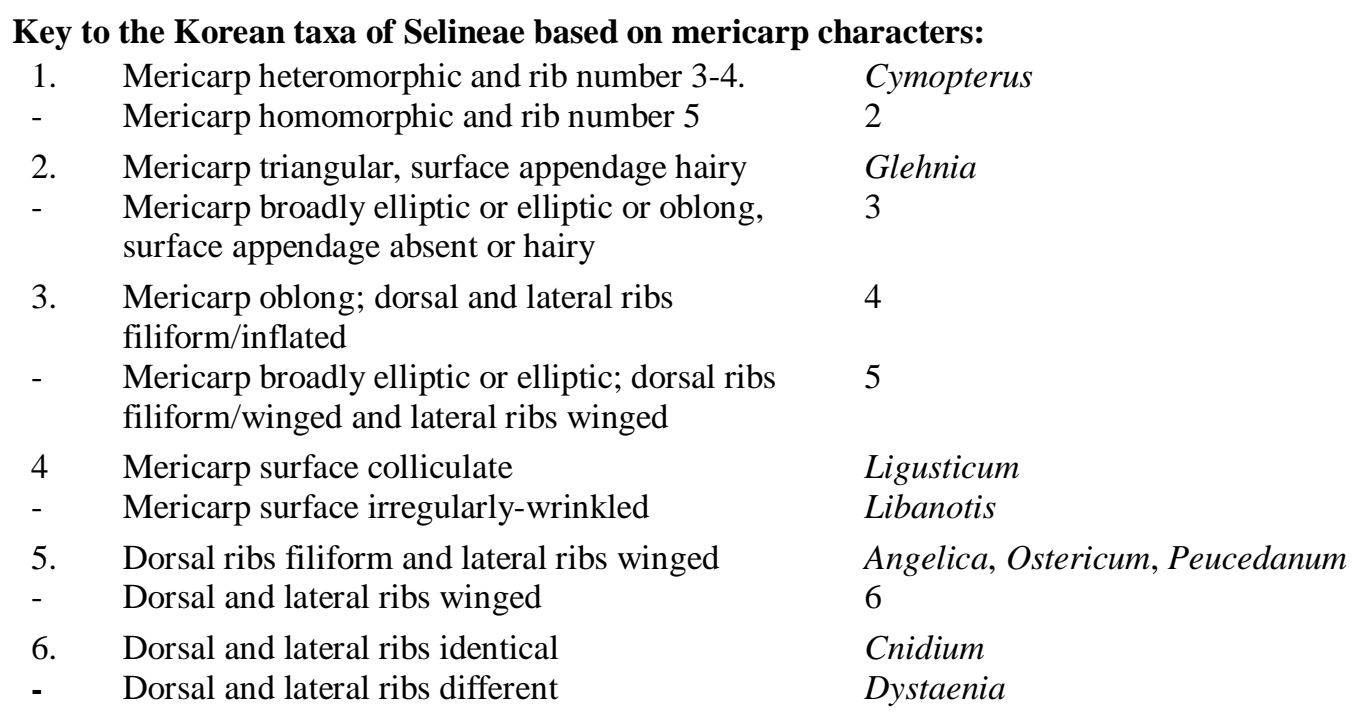

\section{Acknowledgements}

We are grateful to the curator of the herbarium at Korea National Arboretum (KNA), Korea for permitting us to study the voucher specimens. This study was supported by Korea Research Institute of Bioscience and Biotechnology Research Initiative Program of the Republic of Korea. The 3rd (AMD) and 4th (RKC) authors acknowledge to the Memorandum of Understanding between IBMRC, KRIBB and Agharkar Research Institute, India, that facilitated this research.

\section{References}

Ajani, Y., Ajani, A., Cordes, J.M., Watson, M.F. and Downie, S.R. 2008. Phylogenetic analysis of nrDNA ITS sequences reveals relationships within five groups of Iranian Apiaceae subfamily Apioideae. Taxon 57(2): 383-401.

Bagchi, G.D. and Srivastava, G.N. 1989. SEM of epicarp surfaces of some medicinally important Apiaceae. International J. Crude Drug Res. 27(3): 171-177.

Calestani, V. 1905. Contributo alla sistematica delle ombrellifere d'Europa. Webbia 1(1): 89-280.

Choi, H.K., Kim, Y., Sun, B.Y. and Shin, H. 1998. Phylogeny of Dystaenia in subfamily Apioideae (Family Apiaceae) based on ITS sequences. Korean J. Plant Taxon. 28(2): 139-149.

Cronquist, A. 1982. Reduction of Pseudotaenidia to Taenidia (Apiaceae). Brittonia 34: 365-367.

Davis, P.H. 1972. Umbelliferae. In: Davis, P.H. (Ed.), Flora of Turkey and the East Aegean Islands, Vol. 4. Edinburgh University Press, Edinburgh, pp. 265-538.

Downie, S., Ramanath, S., Katz-Downie, D. and Llanas, E. 1998. Molecular systematics of Apiaceae subfamily Apioideae: phylogenetic analyses of nuclear ribosomal DNA internal transcribed spacer and plastid rpo C1 intron sequences. Am. J. Bot. 85: 563-591.

Downie, S.R., Plunkett, G.M., Watson, M.F., Spalik, K., Katz, D., Valiejo, C., Terentieva, E.I., Troitsky, A.V., Lahham, J. and El, A. 2001. Tribes and clades within Apiaceae subfamily Apioideae: the contribution of molecular data. Edinburgh J. Bot. 58: 301-330.

Drude, C.G.O. 1898. Umbelliferae. In: Engelmann, W. (Ed.), Die Natürlichen Pflanzenfamilien, Vol. 3. Leipzig, Germany, pp. 63-250.

Feng, T., Downie, S.R., Yu, Y., Zhang, X., Chen, W., He, X. and Liu, S. 2009. Molecular systematics of Angelica and allied genera (Apiaceae) from the Hengduan Mountains of China based on nrDNA ITS sequences: phylogenetic affinities and biogeographic implications. J. Plant Res. 122: 403-414. 
Hammer, Ø., Harper, D.A.T. and Ryan, P.D. 2001. PAST: Paleontological statistics software package for education and data analysis. Palaeontol. Electronica 4: 1-9.

Harris, J.G. and Harris, M.W. 1994. Plant identification terminology: An illustrated glossary (No. QK9 H37 2001). Spring Lake Publishing, Spring Lake, Utah, pp. 1-216.

Heywood, V.H. 1971. Systematic survery of Old World Umbelliferae. In: Heywood, V.H. (Ed.), The Biology and Chemistry of the Umbelliferae. Academic Press, London, pp. 31-41.

Kondo, K., Terabayashi, S., Okada, M., Yuan, C. and He, S. 1996. Phylogenetic relationship of medicinally important Cnidium offcinale and Japanese Apiaceae based on $r b c$ L sequences. J. Plant Res. 109: 21-27.

Koo, J.C. and Kim, M.Y. 2008. Taxonomic review for the Cyrnopterus melanotilingia (H. Boissieu) C.Y. Yoon in Apiaceae. Korean J. Pl. Taxon. 38: 345-358.

Koso-Poljansky, B.M. 1916. Sciado phytorum systematis lineamenta. Bull. Soc. Imp. Nat. Moscou 29: 93 222.

Lee, E.I, Ko, Y.O., Choi, C.H., Lee, J.K. and Kim, S.H. 1997. Morphological characteristics of seeds in medicinal plants of Umbelliferae. Korean J. Crop Sci. 42(4): 429-434.

Lee, B.Y., Levin, G.A. and Downie, S.R. 2001. Relationships within the spiny-fruited umbellifers (Scandiceae: subtribes Daucinae and Torilidinae) as assessed by phylogenetic analysis of morphological characters. Syst. Bot. 26(3): 622-642.

Liao, C., Downie, S.R., Li, Q., Yu, Y., He, X. and Zhou, B. 2013. New insights into the phylogeny of Angelica and its allies (Apiaceae) with emphasis on East Asian species, inferred from nrDNA, cpDNA, and morphological evidence. Syst. Bot. 38: 266-281.

Liu, M.R., Plunkett, G.M., Lowry, P.P., Van Wyk, B.E. and Tilney, P.M. 2006. The taxonomic value of fruit wing types in the order Apiales. Am. J. Bot. 93: 1357-1368.

Pimenov, M.G. and Leonov, M.V. 1993. The Genera of the Umbelliferae: A Nomenclator. Royal Botanic Gardens, Kew, London, pp. 1-156.

Plunkett, G.M., Soltis, D.E. and Soltis, P.S. 1996. Higher level relationships of Apiales (Apiaceae and Araliaceae) based on phylogenetic analysis of $r b c$ L sequences. Am. J. Bot. 83: 499-515.

Quinn, G.P. and Keough, M.J. 2002. Experimental Design and Data Analysis for Biologists. Cambridge University Press, Cambridge, UK.

Spalik, K., Reduron, J.P. andDownie, S.R. 2004. The phylogenetic position of Peucedanum sensu lato and allied genera and their placement in tribe Selineae (Apiaceae, subfamily Apioideae). Plant Syst. Evol. 243: $189-210$.

Spalik, K., Wojewódzka, A. and Downie, S.R. 2001. The evolution of fruit in Scandiceae subtribe Scandicinae (Apiaceae). Can. J. Bot. 79: 1358-1374.

Stearn, W.T. 1992. Botanical Latin. David and Charles Publishers, London, pp. 489-491.

Swofford, D.L. 2001. Paup*: Phylogenetic analysis using parsimony (and other methods) 4.0. B5.

The Plant List 2013. Version 1.1. Published on the Internet; http://www.theplantlist.org/ (accessed 1st January 2018).

Webb, C.J. and Simpson, M.J. 2001. Seeds of New Zealand gymnosperms and dicotyledons. Manuka Press, pp. $1-428$.

Yoon, C.Y. 2001. Taxonomic position of Cymopterus melanotiligia (H. Boissieu) C.Y. Yoon based on morphological and anatomical characters. Korean J. Pl. Taxon. 31: 253-265.

Zhou, J., Gong, X., Downie, S.R. and Peng, H. 2009. Towards a more robust molecular phylogeny of Chinese Apiaceae subfamily Apioideae: Additional evidence from nrDNA ITS and cpDNA intron (rpl16 and rps16) sequences. Mol. Biol. Evol. 53: 56-68. 